\title{
Novel SNX13 Frameshift Variant in an Individual with Developmental Delay
}

\author{
Xicheng Tao ${ }^{\mathrm{a}}$ Yueping Che ${ }^{\mathrm{a}}$ Chenxi Li $^{\mathrm{a}}$ Wencong Ruan $^{\mathrm{a}}$ Jialu Xu \\ Yonglin $\mathrm{Yu}^{\mathrm{a}}$ Fan Yang ${ }^{\mathrm{b}}$ Jia Wang ${ }^{\mathrm{b}}$ Haifeng $\mathrm{Li}^{\mathrm{a}}$ \\ aDepartment of Rehabilitation, The Children's Hospital, Zhejiang University School of Medicine, Hangzhou, China; \\ ${ }^{\mathrm{b}}$ Cipher Gene, LLC, Beijing, China
}

\section{Established Facts}

- The sorting nexin (SNX) family of proteins plays multiple functions in protein trafficking and intracellular signaling.

- SNXs are associated with several disorders, such as Alzheimer disease and Down syndrome.

\section{Novel Insights}

- No SNX13 gene variants have been found in patients with developmental delays.

- This study provides a new candidate gene for developmental delay.

\section{Keywords}

Global developmental delay · Intellectual disability · SNX13 · Frameshift

\begin{abstract}
Recently, an increasing number of genes have been associated with global developmental delay (GDD) and intellectual disability (ID). The sorting nexin (SNX) protein family plays multiple roles in protein trafficking and intracellular signaling. SNXs have been reported to be associated with several disorders, including Alzheimer disease and Down syndrome. Despite the growing evidence of an association of SNXs with neurodegeneration, SNX13 deficiency has not been associated with GDD or ID. In this study, we present the case of a 4-year-old boy with brain dysplasia and GDD, including lan-
\end{abstract}

karger@karger.com www.karger.com/cgr

Karger $\stackrel{\text { ' }}{5}$

BOPEN ACCESS
C 2021 The Author(s).

Published by S. Karger AG, Basel

This article is licensed under the Creative Commons Attribution 4.0 International License (CC BY) (http://www.karger.com/Services/ OpenAccessLicense). Usage, derivative works and distribution are permitted provided that proper credit is given to the author and the original publisher. guage delay, cognitive delay, and dyskinesia. Exome sequencing revealed a 1-bp homozygous deletion in SNX13 (NM_015132.5: exon8: c.742_743del; p.Tyr248Leufs*20), which caused a frameshift and predicted early termination. Sanger sequencing confirmed that the variant was inherited from his parents respectively. Our findings associate SNX13 variation with GDD for the first time and provide a new GDD candidate gene.

(c) 2021 The Author(s)

Published by S. Karger AG, Basel

\section{Introduction}

Sorting nexin 13 (SNX13) encodes a protein containing $\mathrm{PX}$-associated $(\mathrm{PHOX})$ and regulator of $\mathrm{G}$ protein signaling (RGS) domains, which belongs to the SNX and 
Fig. 1. Cranial MRI of the patient. a T1WI cross section. b T1WI sagittal plane. c T1WI cross section. d FLAIR cross section.
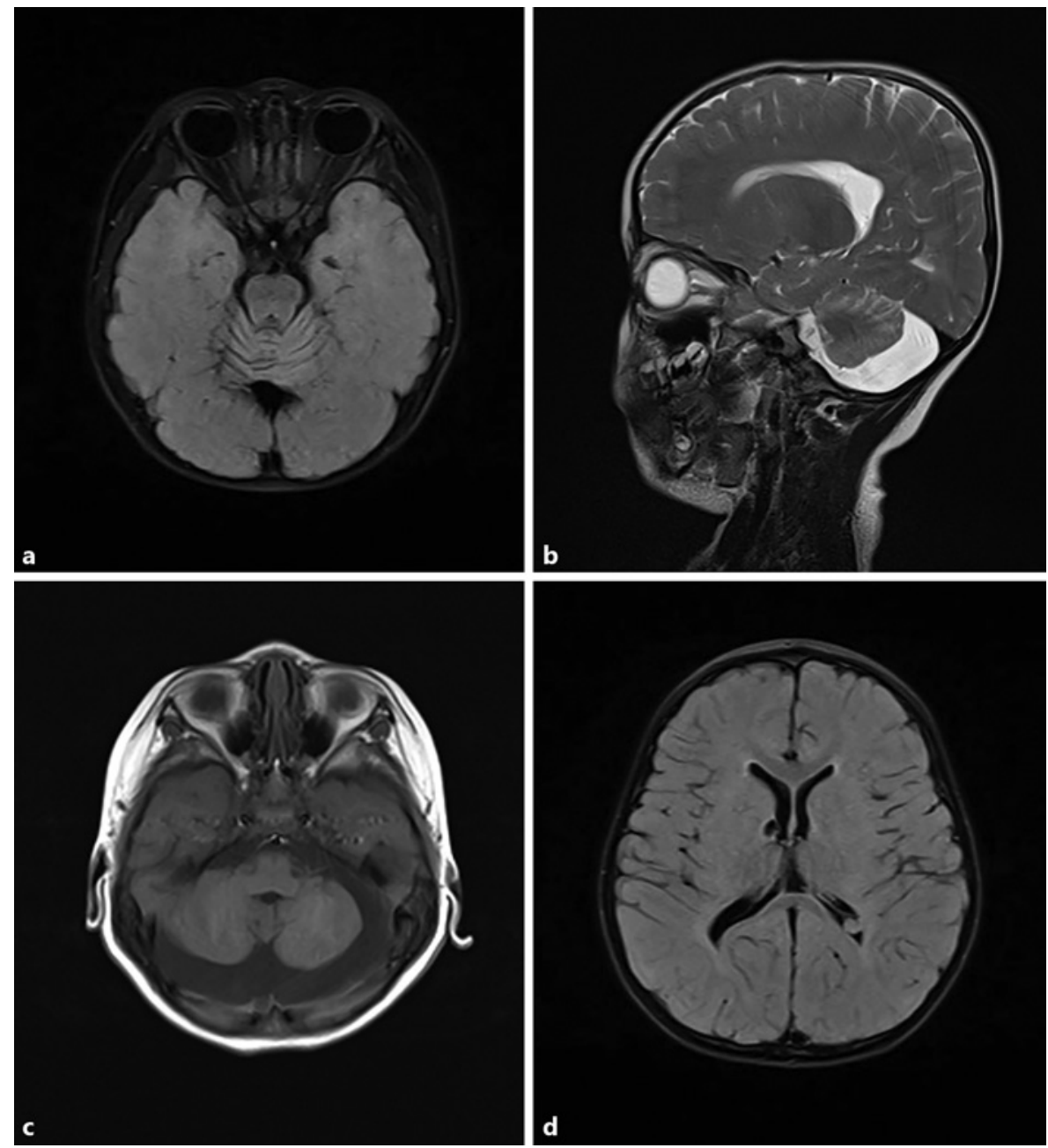

RGS families, respectively. The PHOX domain is a phosphoinositide-binding domain possibly related to the intracellular trafficking of SNX family members, as reported previously [Zheng et al., 2001]. RGS proteins are key modulators of the amplitude and duration of $G$ proteinmediated signaling through their ability to serve as GTPactivating proteins [Zheng et al., 2006]. SNX13 may link heterotrimeric $\mathrm{G}$ protein signaling and vesicular trafficking via the bifunctional role conferred by the PHOX and RGS domains.

Biochemical and cell culture studies have shown that SNX13 attenuates Gas-mediated signaling through its RGS domain and regulates endocytic trafficking and degradation of the epidermal growth factor receptor. A previous study generated an Snx13-null mouse model to investigate the functions of SNX13 in vivo. The Snx13-null embryos exhibited significant overall growth retardation and defects in neural tube closure, blood vessel formation, and the formation of the placental labyrinthine layer [Zheng et al., 2006]. In addition, the Snx13-null visceral yolk sac endoderm cells exhibited dramatic changes in the organization of endocytic compartments, abundant autophagic vacuoles, and abnormal localization of several endocytic markers. All these results suggest that Snx13-null embryos feature defective nutrient uptake and transport, which may contribute to the other developmental abnormalities observed. In this study, we uncovered a new variation in SNX13 in a patient with intellectual disability (ID) and developmental delay (DD) that was inherited from his parents. It was found and confirmed by whole-exome sequencing and Sanger sequencing. Our study found a new candidate gene for DD and expands the mutantion spectrum of SNX13. 


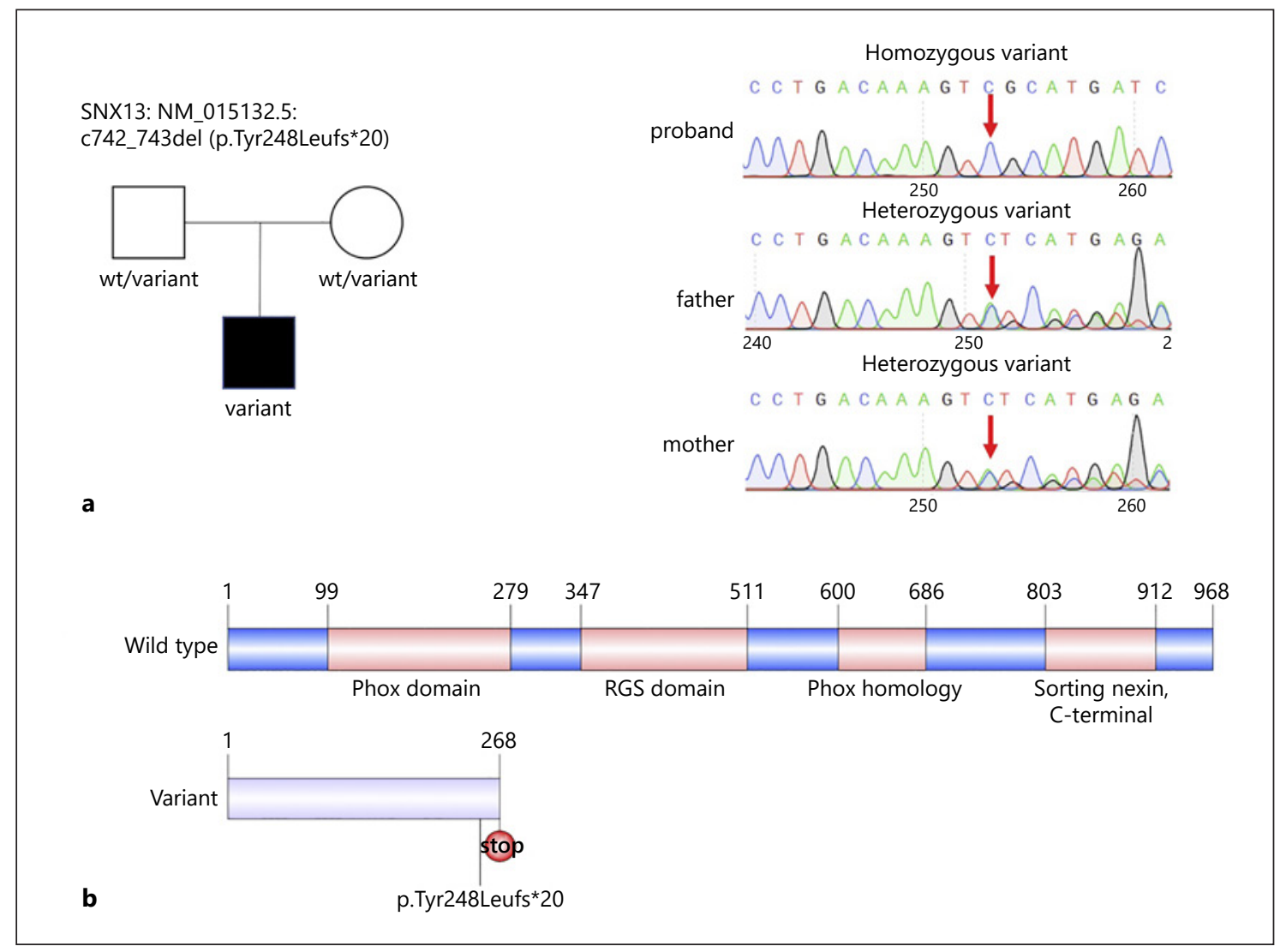

Fig. 2. The homozygous variant in $S N X 13$ was confirmed by Sanger sequencing. a Pedigree chart with $S N X 13$ variant (NM_015132.5: c.742_743del). The homozygous variant was confirmed in the proband and the heterozygous variant was confirmed in the parents by Sanger sequencing. $\mathbf{b}$ The deletion site and variant model for SNX13. The truncated protein lost around 70\% of its sequence and 3 domains compared with full-length SNX13.

\section{Case Report}

\section{Patient}

The 4-year-old patient was the second boy in his family to be delivered spontaneously. His mother took antipsychotic drugs during pregnancy. The patient exhibited developmental regression manifested as poor voice tracking, unconscious vocalizations, and the inability to actively grasp objects with his hands, hold his head firmly, or roll over at 7 months old. Thus far, rehabilitation treatment has had no obvious effect.

\section{Clinical Features}

Further physical examination revealed reduced extremity muscle tone (clinical grade 1), extremity muscle strength (grade IV), negative bilateral patellar tendon reflex, negative Pap sign, and negative ankle clonus. Cranial magnetic resonance imaging (MRI) (Fig. 1) indicated cerebellar volume reduction, dysplasia, an occipital arachnoid cyst, and slightly enlarged abnormal signal nodules near the right lateral ventricle. White matter degeneration of bilateral lateral ventricles was also observed. No obvious abnormalities were observed in routine examinations of serum ammonia $(53 \mu \mathrm{mol} / \mathrm{L}$ ), genetic metabolism A + B, TORCH (Toxoplasmosis,
Other agents, Rubella, Cytomegalovirus, Herpes virus), thyroid function test, routine blood C-reactive protein (CRP), urine, stool, biochemistry, coagulation profile, blood gas, electrolytes, lactic acid, and immune detection.

\section{Follow-up}

Development of the proband was evaluated when he was 4 years and 2 months old. Evaluation revealed a mental development index score (MI) $<50$ (equal to average child aged 6-7 months), Daring intelligence quotient (DQ) development index $<50$ (equal to average child aged $7-8$ months), and gross motor ability (GMFM-88) total score of 32.9 (on the scale for children with cerebral palsy, Gross Motor Function Classification System [GMFCS] classification of grade IV). Presently, he can use gestures to express his ideas, initiatively grasp objects, roll over, and sit stably. However, he exhibits unconscious pronunciation and poor fine function development, coordination, climbing, unassisted walking, and response to name calling.

\section{Gene Testing}

Whole-exome sequencing (WES) was performed to further clarify the cause of the phenotype. Written informed consent was 
obtained from the proband's parents. Raw data were mapped to the human reference genome hg38 using the Burrows-Wheeler Aligner (BWA) [Abuin et al., 2015]. Variant calling was performed by Genome Analysis Toolkit (GATK) [McKenna et al., 2010], and annotation was conducted using ANNOVAR including population databases (ESP6500, 1000 Genomes, Exome Aggregation Consortium [ExAC], gnomAD), published or submitted variants (HGMD, Clinvar), and in silico pathogenicity predictions for missense variants (SIFT, PolyPhen2, LRT, MutationTaster, FATHMM, CADD, REVEL) and for splice site variants (MaxEntScan, NNSplice, dbscSNV) [Wang et al., 2010]. The criteria for variant filtering were as follows:

1. Variants located in exon and splicing $( \pm 20 \mathrm{bp})$ region and minor allele frequency $<0.005$ for gnomAD_exome_popmax, gnomAD_genome_popmax, and gnomAD3_genome_AF_ Popmax were selected (online suppl. Table 1; see www. karger.com/doi/10.1159/000520296).

2 . Then variants were classified according to inheritance pattern: de novo variants, autosomal recessive (AR) inheritance of homozygous variants, AR inheritance of compound heterozygous variants, X-linked inheritance (online suppl. Table 1).

Variants meeting the inheritance pattern were evaluated based on population frequency and pathogenicity prediction. Finally, the homozygous variant NM_015132.5: exon8: c.742_743del (p.Tyr248Leufs*20) of SNX13 inherited form both parents was identified as a candidate causative variant for DD in our patient by clinical phenotype association and literature research. This variant caused a frameshift and resulted in a truncated protein (268 amino acids), which lost about $70 \%$ sequences compared with the fulllength SNX13 (Fig. 2b). The variant was not reported in the public databases (online suppl. Table 1), indicating the rarity of this mutation. No further pathogenic variants related to this phenotype were identified.

Exome-based copy number variant (CNV) calling [Zhai et al., 2021] for WES data was performed to identify small insertions/ deletions (indels). CNVs were detected in the WES data using ExomeDepth. Meanwhile, CNV sequencing (CNV-seq) was used for validation. Genomic DNA was fragmented, and sequencing libraries were prepared using the TruSeq Library Construction Kit. Libraries were sequenced using a high-throughput sequencing platform (Illumina, San Diego, CA, USA). All sequences were aligned to the human reference genome hg38 using the Burrows-Wheeler algorithm [Li and Durbin, 2009]. All candidate CNVs detected from WES data or CNV-seq were filtered through the Database of Genomic Variants (http://dgv.tcag.ca). CNVs were annotated using literature reviews and public databases (Decipher, ClinVar, ClinGen, ISCA, and dbVar), and pathogenicity screening was performed using OMIM, DECIPHER, Orphanet, and other databases. No CNVs or other structural variants were found that could explain the patient's phenotype.

The homozygous variant of the proband and heterozygous genotypes of his parents were confirmed by Sanger sequencing, which suggested that the homozygous variant was inherited from the parents (Fig. 2a). It was noted that the parents who carried the same heterozygous variant, were not close relatives and did not have global DD (GDD) symptoms in their families. Moreover, no other homozygous, X-linked, or de novo variants that could explain the phenotype of the patient were identified according to the minor allele frequency, inheritance pattern matching, and literature investigation (online suppl. Table 1).

SNX13 Variation with Development Delay

\section{Discussion}

Although SNX protein family members have been regarded as regulators of cargo transport, additional studies have expanded our knowledge of their role. For example, $\operatorname{Snx} 4 \mathrm{p}$ was found to play a crucial role in regulating cytoplasm-to-vacuole protein targeting and autophagy [Scott et al., 1996]. SNX23 (KIF16B) was found to transport early endosomes to the plus end of microtubules, modulate the intracellular localization of early endosomes, and balance receptor recycling and degradation [Hoepfner et al., 2005]. SNX13, SNX14, SNX19, and SNX25, exhibit a unique architecture consisting of a central Phox-homology (PX) domain flanked by several conserved domains belonging to the PXA-RGS-PX-PXC subfamily [Teasdale and Collins, 2012].

Few genetic diseases have been reported to be related to gene variations in this subfamily. SNX19 was reported as a candidate gene for Jacobsen syndrome, which is characterized by DD and ID [Ji et al., 2010]. Furthermore, patients with distinctive AR cerebellar ataxia and ID syndrome were found to have SNX14 variations [Thomas et al., 2014]. SNX14 was predicted to be highly coexpressed with genes involved in cellular protein metabolism and vesicle-mediated transport. Functional verification showed that SNX14 deficiency resulted in increased cytoplasmic vacuolation, which is observed in cultured fibroblasts.

Domain information usually indicates the possible roles of a protein. SNX13 has 2 transmembrane domains, a PHOX domain, an RGS domain, a PX domain, and a C-terminal PX-associated domain. The RGS domain has been reported as a GTPase activating protein and accelerates GTP hydrolysis in the $\mathrm{G}$ protein $\alpha$ subunit, resulting in the termination of $\mathrm{G}$ protein-coupled receptor signaling [Zheng et al., 2001]. The PX domain has a more extensive role and is associated with various intracellular proteins; more importantly, it selectively interacts with phosphatidylinositol-3-phosphate and other phosphoinositides [Amatya et al., 2021].

The variant in our case was predicted to result in a translation stop near the end of the PHOX domain, and a total loss of the other 3 domains. Therefore, the expressed protein may lose the ability to activate GTPase and communication with various intracellular molecules. A gene-targeting construct was assembled to allow the excision of coding exon 3 in Snx13-null mice that is expected to generate a translational frameshift mutation and a product containing only the $\mathrm{N}$-terminal 42 residues of SNX13 [Zheng et al., 2006]. Snx13-null 
mice were embryonic lethal around midgestation, possibly because of the premature termination of translation which produced a shorter protein lacking all functional domains. The Snx13-null mice exhibited growth retardation and abnormal brain development (including failure of neural tube closure and cephalic vascularization), consistent with observations in our patient. The cranial MRI of our patient revealed cerebellar volume reduction, dysplasia, and an occipital arachnoid cyst (Fig. 1). But there have been different outcomes in the 2 species.

The diverse species-specific phenotypic consequences of loss-of-function mutations are not uncommon; this was also observed in investigations of SNX14 [Bryant et al., 2020]. SNX14 belongs to the RGS-PX protein family and contains conserved functional domains similar to those of SNX13. Variants of SNX14 were found in individuals with autosomal recessive spinocerebellar ataxia 20 (SCAR20; OMIM 616354), who presented with clinical features similar to those of our patient. Surprisingly, homozygous Snx14 knockout mice are embryonic lethal, but the loss-of-function mutation in SNX14 was viable in other species, including humans [Thomas et al., 2014], dogs [Fenn et al., 2016], zebrafish [Bryant et al., 2020], Drosophila [Suh et al., 2008], and yeast [Henne et al., 2015].

This interspecies variation occurs despite evidence that the RGS-PX protein family has a conserved biochemical role in lipid biogenesis across species ranging from humans to yeast [Teasdale and Collins, 2012]. Both Snx13 and $\operatorname{Sn} x 14$ knockout mice fail during embryonic development, mainly due to placentation defects, and these phenotypes have recently been identified as the principal cause of embryonic lethality in mouse mutants [Mohun et al., 2013]. We speculate that SNX13 and SNX14 play a more critical role in early embryonic development in mice than in other species; therefore, mutations have differential effects in different species.

\section{Conclusion}

In summary, SNX13 and SNX14 have similar functions in inter-organelle communication [Thomas et al., 2014], and the deficiency may affect the synaptic function, which has a crucial role in neurodevelopmental disorders [Zoghbi and Bear, 2012]. Therefore, SNX13 may play an important role in neurodevelopment. Here, we report the first case of GDD in a patient exhibiting an SNX13 variant and provide a new candidate gene for
GDD. Future studies will aim to understand the underlying molecular mechanisms involved in SNX13 function.

\section{Acknowledgments}

We thank the proband and his family for their active collaboration and Cipher Gene LLC for sequencing and data mining.

\section{Statement of Ethics}

Research was conducted ethically in accordance with the World Medical Association Declaration of Helsinki. This study protocol was reviewed and approved by the local Research Ethics Committee of The Children's Hospital, Zhejiang University School of Medicine. Written informed consent was obtained from the patient's parents for publication of this case report and any accompanying images.

\section{Conflict of Interest Statement}

The authors have no conflicts of interest to declare.

\section{Funding Sources}

This study was supported by the multi-center clinical research project in Zhejiang Province (grant No. S20A0002). The sponsors had no role in the design, methods, subject recruitment, data collection, analysis, or preparation of the manuscript.

\section{Author Contributions}

X.T. and Y.C.: conceptualization, methodology, manuscript preparation; C.L., W.R., J.X., and Y.Y.: clinical data, manuscript preparation; F.Y. and J.W.: software, data mining; H.L.: supervision, writing, reviewing, and editing. All authors read and approved the final manuscript.

\section{Data Availability Statement}

The data that support the findings of this study are available on request from the corresponding author. 


\section{References}

Abuin JM, Pichel JC, Pena TF, Amigo J. BigBWA: approaching the Burrows-Wheeler aligner to Big Data technologies. Bioinformatics. 2015; 31:4003-5.

Amatya B, Lee H, Asico LD, Konkalmatt P, Armando I, Felder RA, et al. SNX-PXA-RGSPXC Subfamily of SNXs in the Regulation of Receptor-Mediated Signaling and Membrane Trafficking. Int J Mol Sci. 2021;22:2319.

Bryant D, Seda M, Peskett E, Maurer C, Pomeranz G, Ghosh M, et al. Diverse species-specific phenotypic consequences of loss of function sorting nexin 14 mutations. Sci Rep. 2020;10: 13763.

Fenn J, Boursnell M, Hitti RJ, Jenkins CA, Terry RL, Priestnall SL, et al. Genome sequencing reveals a splice donor site mutation in the SNX14 gene associated with a novel cerebellar cortical degeneration in the Hungarian Vizsla dog breed. BMC Genet. 2016;17:123.

Henne WM, Zhu L, Balogi Z, Stefan C, Pleiss JA, Emr SD. Mdm1/Snx13 is a novel ER-endolysosomal interorganelle tethering protein. J Cell Biol. 2015;210:541-51.

Hoepfner S, Severin F, Cabezas A, Habermann B, Runge A, Gillooly D, et al. Modulation of receptor recycling and degradation by the endosomal kinesin KIF16B. Cell. 2005;121:437-50.
Ji T, Wu Y, Wang H, Wang J, Jiang Y. Diagnosis and fine mapping of a deletion in distal 11q in two Chinese patients with developmental delay. J Hum Genet. 2010;55:486-9.

Li H, Durbin R. Fast and accurate short read alignment with Burrows-Wheeler transform. Bioinformatics. 2009;25:1754-60.

McKenna A, Hanna M, Banks E, Sivachenko A, Cibulskis K, Kernytsky A, et al. The Genome Analysis Toolkit: a MapReduce framework for analyzing next-generation DNA sequencing data. Genome Res. 2010;20:1297-303.

Mohun T, Adams DJ, Baldock R, Bhattacharya S, Copp AJ, Hemberger M, et al. Deciphering the Mechanisms of Developmental Disorders (DMDD): a new programme for phenotyping embryonic lethal mice. Dis Model Mech. 2013;6:562-6.

Scott SV, Hefner-Gravink A, Morano KA, Noda T, Ohsumi Y, Klionsky DJ. Cytoplasm-tovacuole targeting and autophagy employ the same machinery to deliver proteins to the yeast vacuole. Proc Natl Acad Sci U S A. 1996; 93:12304-8.

Suh JM, Stenesen D, Peters JM, Inoue A, Cade A, Graff JM. An RGS-containing sorting nexin controls Drosophila lifespan. PLoS One. 2008;3:e2152.

Teasdale RD, Collins BM. Insights into the PX (phox-homology) domain and SNX (sorting nexin) protein families: structures, functions and roles in disease. Biochem J. 2012;441:39_ 59.
Thomas AC, Williams H, Setó-Salvia N, Bacchelli C, Jenkins D, O'Sullivan M, et al. Mutations in SNX14 cause a distinctive autosomal-recessive cerebellar ataxia and intellectual disability syndrome. Am J Hum Genet. 2014;95: 611-21.

Wang $\mathrm{K}$, Li M, Hakonarson H. ANNOVAR: functional annotation of genetic variants from high-throughput sequencing data. $\mathrm{Nu}$ cleic Acids Res. 2010;38:e164.

Zhai Y, Zhang Z, Shi P, Martin DM, Kong X. Incorporation of exome-based $\mathrm{CNV}$ analysis makes trio-WES a more powerful tool for clinical diagnosis in neurodevelopmental disorders: A retrospective study. Hum Mutat. 2021;42:990-1004.

Zheng B, Ma YC, Ostrom RS, Lavoie C, Gill GN, Insel PA, et al. RGS-PX1, a GAP for GalphaS and sorting nexin in vesicular trafficking. Science. 2001;294:1939-42.

Zheng B, Tang T, Tang N, Kudlicka K, Ohtsubo $\mathrm{K}, \mathrm{Ma} \mathrm{P}$, et al. Essential role of RGS-PX1/sorting nexin 13 in mouse development and regulation of endocytosis dynamics. Proc Nat Acad Sci U S A. 2006;103:16776-81.

Zoghbi HY, Bear MF. Synaptic dysfunction in neurodevelopmental disorders associated with autism and intellectual disabilities. Cold Spring Harb Perspect Biol. 2012;4:a009886. 